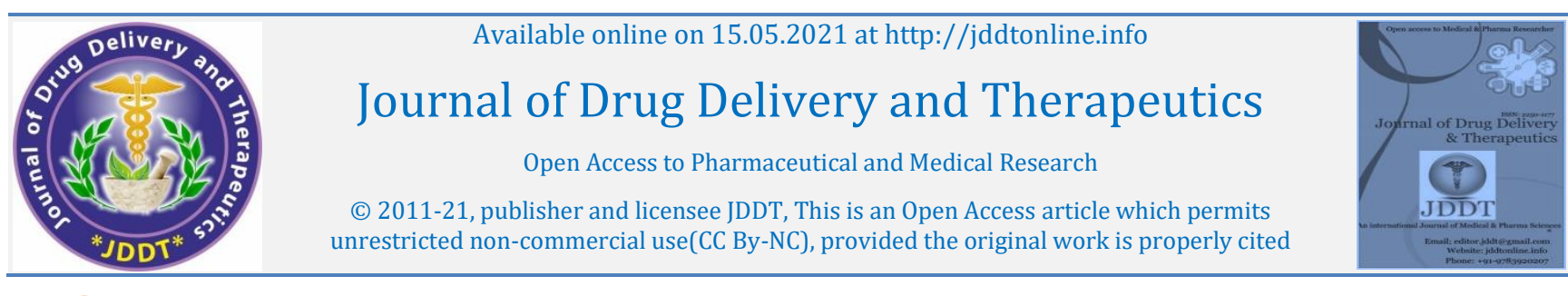

Open Access Full Text Article

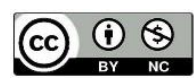

Case Report

\title{
Male genital allergy with penile oedema and severe aseptic urethritis, due to vulvovaginal candidiasis, simulating sexually transmitted infection
}

\author{
Kamel El-Reshaid *, Hosameldin Tawfik Sallam ** \\ * Department of Medicine, Faculty of Medicine, Kuwait University, P O Box 24923, 13110 Safat, Kuwait \\ ** Department of Medicine, Amiri hospital, Ministry of Health, Kuwait
}

Article Info:

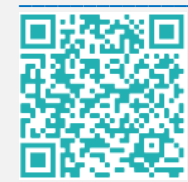

\section{Article History:}

Received 21 March 2021

Review Completed 16 April 2021

Accepted 12 April 2021

Available online 15 May 2021

*Address for Correspondence:

Dr. Kamel El-Reshaid, Professor, Dept. Of Medicine,

Faculty of Medicine, Kuwait University,P O Box 24923, 13110 Safat, Kuwait

\begin{abstract}
Sexually transmitted diseases (STDs) are frequently encountered and more than 1 million are acquired daily. Genital allergy (GA), on the other hand, is another uncommon cause of sexually transmitted disorders that should be considered if no infection is identified or genital soreness persists or worsens with antimicrobial therapy. In this case report; we present a patient, with severe GA (type I hypersensitivity reaction) to vulvovaginal candidiasis in his sex partner. He was initially misdiagnosed as STD as he presented with severe penile and urethral oedema culminating in hematuria and bladder neck obstruction due to severe urethral oedema. Our systematic approach in diagnosis and hence treatment of the patient and his sex partner is outlined.
\end{abstract}

Keywords: Candidiasis, genital allergy, penile oedema, STD, urethritis.

Cite this article as:

El-Reshaid K, Sallam HT, Male genital allergy with penile oedema and severe aseptic urethritis, due to vulvovaginal candidiasis, simulating sexually transmitted infection, Journal of Drug Delivery and Therapeutics. 2021; 11(3):4-6 DOI: http://dx.doi.org/10.22270/jddt.v11i3.4699

\section{INTRODUCTION}

Genital infections, vulvitis and balanitis, are frequently encountered sexually transmitted diseases (STDs) and more than 1 million are acquired daily 1 . STDs are classified as either; (a) gonococcal urethritis, caused by Neisseria gonorrhea, or (b) non-gonococcal urethritis, most commonly caused by chlamydia trachomatis in $20-50 \%$ followed by Mycoplasma genitalium, Trichomonas vaginalis, Adenoviridae, Uropathogenic Escherichia coli, Herpes simplex, Cytomegalovirus, Ureplasma urealyticum, Staphylococcus aureus, Streptococcus group B and Fungal urethritis 2. Genital allergy (GA), on the other hand, is another uncommon cause of sexually transmitted disorders that should be considered if no infection is identified or genital soreness persists or worsens with antimicrobial therapy ${ }^{3}$. In this case report; we present such rare presentation of severe GA with aseptic urethritis and hematuria due to severe urethral oedema and outline its management.

\section{THE CASE:}

A 51-year-old man presented with severe pain, itching and swelling of his genitalia for 2 days following recent sexual encounter with a new partner. He also had expressed difficulty in passing urine with poor flow and bleeding during urination. He denied fever, shortness of breath, oedema, abdominal pain, skin rash and joint pains. The patient did not have past history of significant medical illness, surgery, allergy or chronic intake of medications.
After testing for sexually transmitted diseases; he had received Ciprofloxacin $500 \mathrm{mg}$ twice daily yet without benefit. On his initial physical examination, the patient was conscious and oriented X3. He was in distress of his pain in his genitalia. He was afebrile and blood pressure was 120/80 mm Hg. Systemic examination did not show abnormality. However, examination of the genitalia revealed swollen penis (Figure 1A) and urethra (Figure 2). There was no urethral discharge and groin lymphadenopathy. Laboratory investigations showed normal peripheral leucocytic and platelets counts. Hemoglobin was normal with normal MCV. Serum sugar, urea, creatinine, electrolytes and liver functions were normal. TSH was normal. Serum procalcitonin was normal at $0.02 \mathrm{ug} / \mathrm{L}$. His initial tests for STD showed; (a) hematuria on urine testing without proteinuria and pyuria, (b) negative urinary bacterial culture, (c) negative nucleic acid amplification test (NAAT) for chlamydia trachomatis. Abdominal and pelvic ultrasound did not show abnormality and CT pelvis confirmed normal posterior urethra with normal urinary bladder and prostate (Figure 3). Uroflowmetry showed very low maximal flow rate (Q-max at $8 \mathrm{ml} /$ seconds) with maximal voided urine at $200 \mathrm{ml}$. Moreover, serum IgE level was high $(1000 \mathrm{IU} / \mathrm{ml})$. Hence diagnosis of genital allergy was established. The patient was treated with Solumedrol $500 \mathrm{mg}$ IV daily for 3 days with Diclofenac sodium $75 \mathrm{mg}$ IM 1-2/day for pain relieve. His stomach was protected with Omeperazole $20 \mathrm{mg}$ daily. Within few hours, his penile oedema and urination improved dramatically (Figure 1 B). Subsequently, medications were changed to Prednisone $20 \mathrm{mg}$ daily with 
Omeprazole $20 \mathrm{mg}$ daily for 1 week. Evaluation of his sexual partner revealed vulvovaginal candidiasis without chlamydia trachomatis and urinary tract infection. She received oral Fluconazole $150 \mathrm{mg}$ followed by another dose 3 days later and a topical antifungal cream for 1 week. One month subsequent to such therapy, both partners enjoyed normal sex life without recurrence of GA.

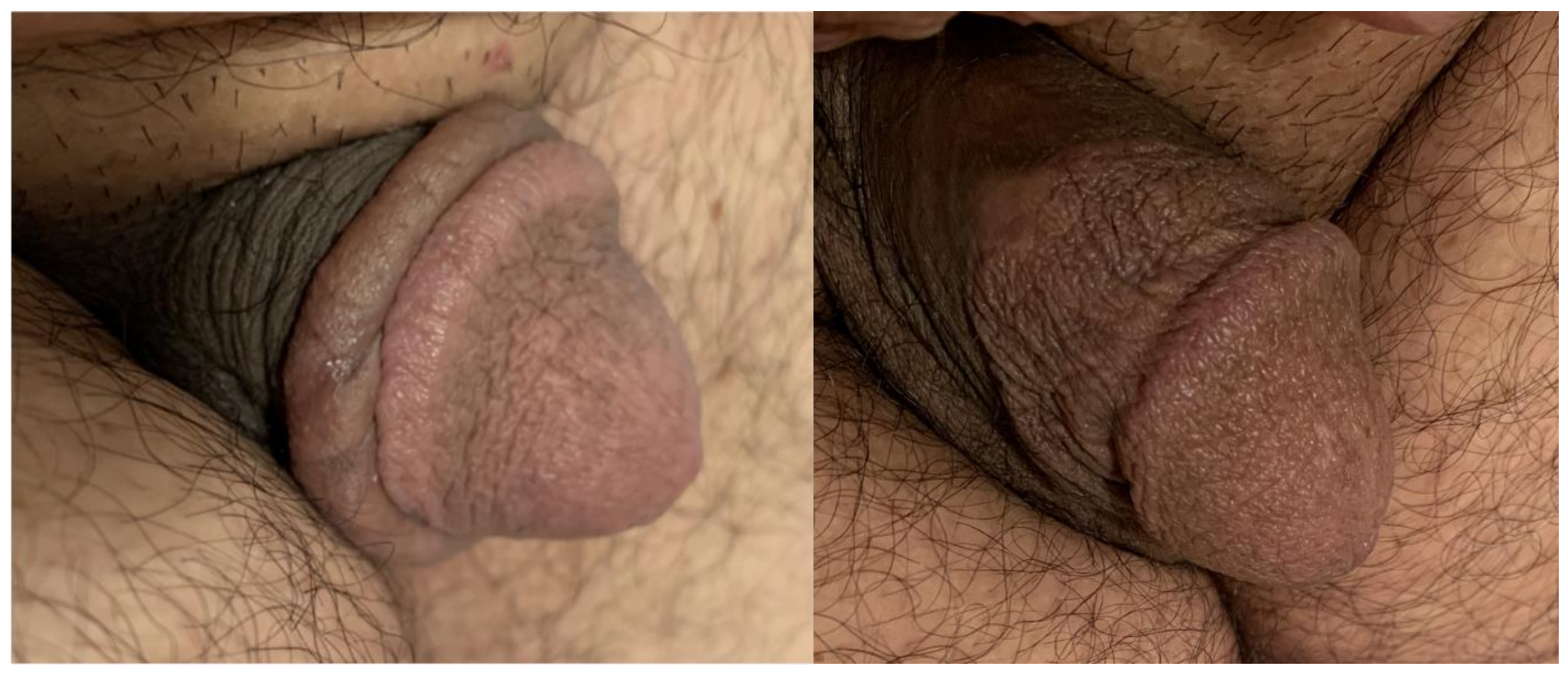

Figure 1: Showing penile oedema at initial presentation (A) and its resolution, few hours later, with IV Solumedrol (B).

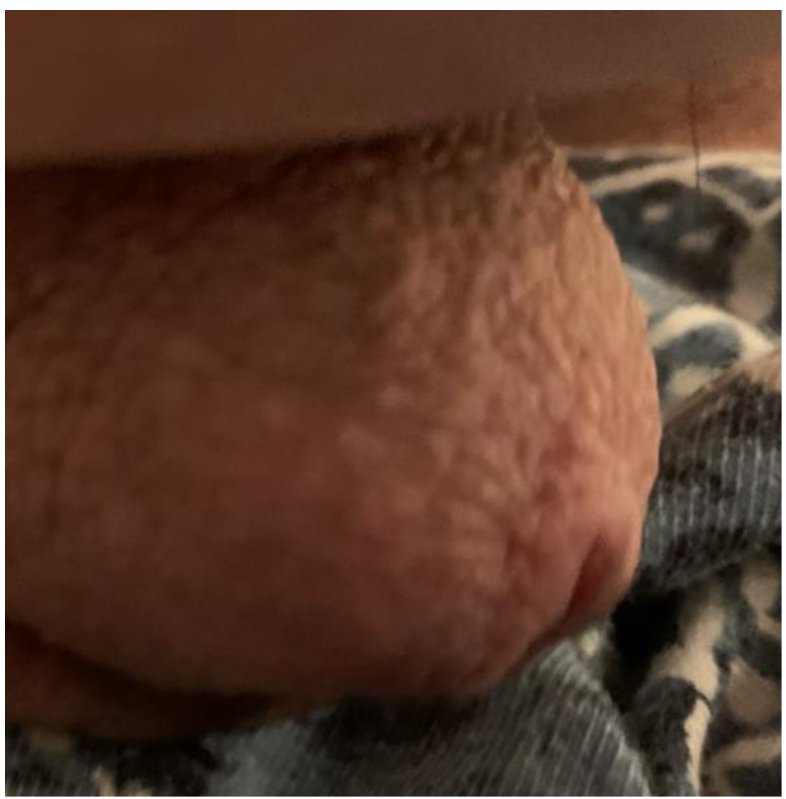

Figure 2: Showing severe urethral oedema at initial presentation.

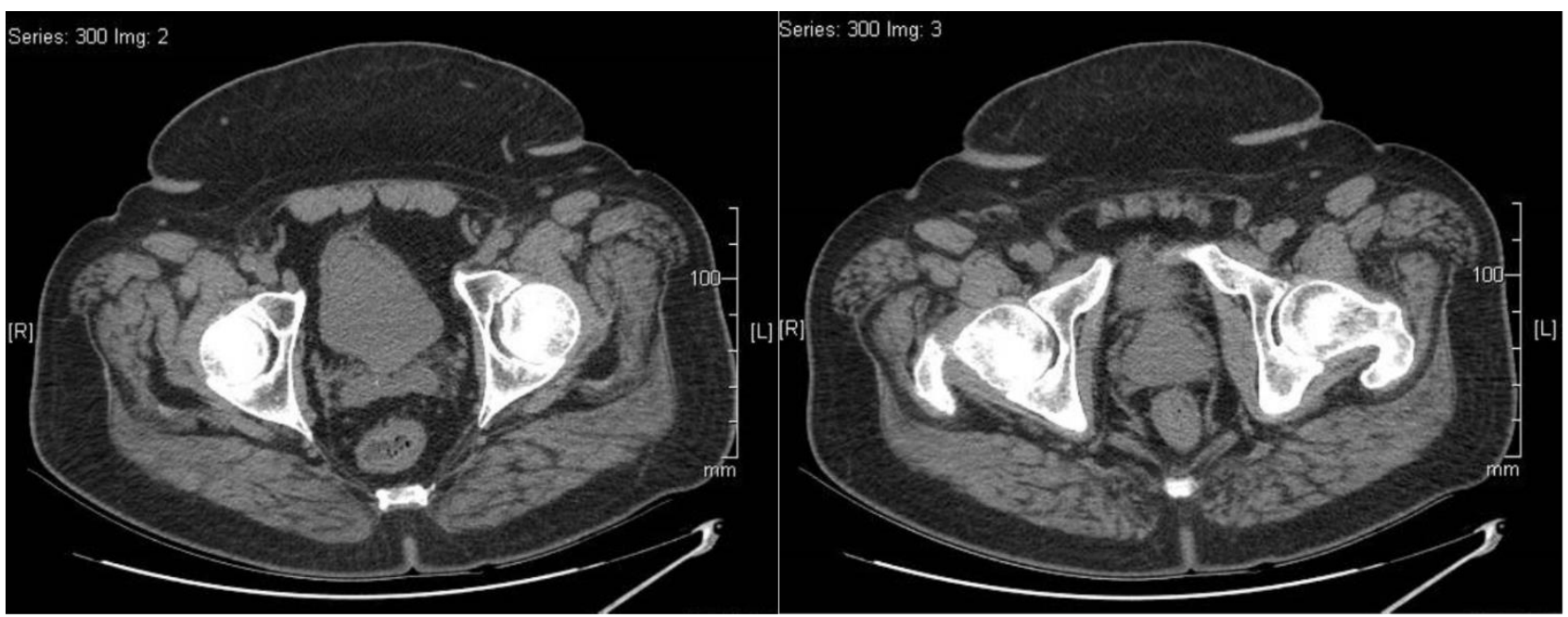

Figure 3: Axial CT scan views, of the patient's pelvis, showing intact urinary bladder (UB) in (A) and normal prostate (P) in (B) ISSN: 2250-1177 


\section{DISCUSSION}

Our patient had severe urethral oedema simulating septic urethritis. However, his GA was disclosed by: (a) severe penile oedema, (b) negative STD testing, (c) high IgE antibodies, (d) normal prostate and bladder by CT scan, and (e) lack of response to antibiotics and dramatic response to corticosteroids. Moreover, the acute post-coital event i.e. type I hypersensitivity reaction without chronic vesicular disease has ruled out endogenous eczema due to chronic and repetitive exposure to local allergens (type IV hypersensitivity) 4 . The reported list of such local genital allergens include; semen, spermicides, condoms-latex, KY jelly lubricants. Moreover, non-sexually related hypersensitivity reactions include topical medications viz. ethylenediamine (present in Triadcortyl), framycetin, neomycin, clobetasol propionate, and crotamiton (Eurax) and rarely imidazole antifungals 5 . Such contact dermatitis may sometimes prove difficult to distinguish clinically (without skin RAST testing) from irritant dermatitis. The list of the latter includes; feminine hygienic spray from fluorinated hydrocarbon propellants, bubble baths, cosmetics and self-adhesive pads for excessive vaginal secretions, urine-leak (ammonia). In our patient; the etiology of his GA is unique. Vulvovaginal candidiasis was the culprit in his sex partner. Candida is a well-recognized allergen. In vitro tests have documented the release of histamine from rat mast cells by candida antigens ${ }^{6}$. Anticandida IgE antibodies are often present in the vaginal secretions of women with recurrent VVC but not in control women ${ }^{7}$. Vulvovaginal candidiasis has been reported to cause bronchial hypersensitivity and even glans soreness in a male partner hours after sexual intercourse with an infected woman ${ }^{8}$. In conclusion; GA simulate STD and should be considered in patients with penile oedema associated with aseptic urethritis and futile antimicrobial therapy.

\section{REFERENCES:}

1- WHO factsheet. WHO. Dec 2015- 2015 Sexually Transmitted Diseases Treatment Guidelines: Diseases Characterized by Urethritis and Cervicitis. Centers for Disease Control and Prevention. Available at https://www.cdc.gov/std/tg2015/urethritis-andcervicitis.htm. June 4, 2015; Accessed: December 18, 2020.

2- Young A, Toncar A, Wray AA. Urethritis. [Updated 2020 Dec 14]. In: StatPearls [Internet]. Treasure Island (FL): StatPearls Publishing; 2021 Jan-. Available from: https://www.ncbi.nlm.nih.gov/books/NBK537282.

3- Sonnex C. Genital allergy. Sex Transm Infect. 2004; 80:4-7.

4- English J. Current concepts of irritant contact dermatitis. Occup Enviro Med. 2004; 61:722-726.

5- Bauer A, Geier J, Elsner P. Allergic contact dermatitis in patients with anogenital complaints. J Reprod Med. 2000; 45:649-654.

6- Nosal R. Histamine release from isolated rat mast cells due to glycoprotein from Candida albicans in vivo. J Hygiene Epidem Microbiol Immunol. 1974; 18:377-8.

7- Fidel PL Jr, Sobel JD. Immunopathogenesis of recurrent vulvovaginal candidiasis. Clin Microbiol Rev. 1996; 9:335-48.

8- Catterrall RD. Urethritis and balanitis due to Candida. In: Winner HI, Hurley R, eds. Symposium on candida infection. London: Livingstone, 113-8. 